\title{
Knockdown of LINC01123 inhibits cell viability, migration and invasion via miR-361-3p/TSPAN1 targeting in cervical cancer
}

\author{
CUI LI, YUJUAN LI, YUYING ZHANG, HUA YAN and CHENGXIANG HUANG \\ Department of Gynecology, Linyi Central Hospital, Linyi, Shandong 276400, P.R. China
}

Received September 7, 2020; Accepted March 29, 2021

DOI: $10.3892 /$ etm.2021.10618

\begin{abstract}
Cervical cancer (CC) is a type of gynecological malignancy that poses a significant threat to females. The aim of the present study was to examine the role of long intergenic non-protein coding RNA 1123 (LINC01123) and its underlying molecular mechanism in the development of CC. mRNA expression levels of LINC01123 and microRNA (miR)-361-3p in CC tissue samples and cell lines were evaluated using reverse transcription-quantitative PCR. Cell viability, migration and invasion were detected using 3-(4,5-dimethylthiazol-2-yl)-2,5-diphenyltetrazolium bromide, wound healing and Transwell assays. Moreover, a xenograft tumor model was established for elucidating the influence of LINC01123 knockdown on tumor growth in vivo. A dual-luciferase reporter assay was used to confirm the association between LINC01123 and miR-361-3p, and miR-361-3p and tetraspanin 1 (TSPAN1). Western blot analysis was used to determine TSPAN1 protein expression. LINC01123 expression was upregulated and miR-361-3p expression was reduced in CC tissue samples and cell lines. Knockdown of LINC01123 inhibited cell viability, migration and invasion in vitro, and suppressed tumor growth in vivo. Furthermore, LINC01123 targeted miR-361-3p and negatively regulated miR-361-3p expression. Overexpression of miR-361-3p inhibited cell viability, migration and invasion in HeLa and CaSki cells. Additionally, miR-361-3p targeted TSPAN1 and negatively regulated TSPAN1 expression. Inhibition of miR-361-3p and overexpression of TSPAN1 reversed the effect of LINC01123 knockdown on cell proliferation, migration and invasion in HeLa cells. Knockdown of LINC01123 inhibited cell proliferation, migration and invasion via miR-361-3p/TSPAN1 regulation in $\mathrm{CC}$, which may present an effective target for treatment of $\mathrm{CC}$.
\end{abstract}

Correspondence to: Dr Chengxiang Huang, Department of Gynecology, Linyi Central Hospital, 17 Health Road, Yishui, Linyi, Shandong 276400, P.R. China

E-mail: huangcx307@126.com

Key words: cervical cancer, long intergenic non-protein coding RNA 1123, microRNA-361-3p, tetraspanin 1, tumorigenesis

\section{Introduction}

Cervical cancer (CC) is a type of gynecological malignancy and a major cause of cancer-related mortality in females worldwide (1). With $\sim 6$ million cases per year, CC continues to be a major global health issue (2). Recently, studies have focused on identifying effective targets that may be useful for screening and treating CC (3). Despite advances in therapeutic strategies, including chemotherapy, surgery, radiation and targeted therapy, the survival rate of patients remains low $(4,5)$. Thus, it is essential to investigate the potential biological mechanism and elucidate novel targets for improving therapeutic strategies for CC.

Long non-coding RNAs (lncRNAs), $>200$ nt without protein-coding capacity are associated with various biological functions (6). Accumulating evidence indicates that lncRNAs play important roles in tumorigenesis, including in CC. LncRNA XLOC_006390 promotes the invasion and migration of CC cells and facilitates $\mathrm{CC}$ tumorigenesis and metastasis (7). LncRNA small nucleolar RNA host gene 1 knockdown inhibits cell viability and migration in HeLa and C-33A cells (8). Recently, long intergenic non-protein coding RNA 1123 (LINC01123) has been reported to exhibit a carcinogenic function in certain types of cancer. For example, LINC01123 enhances cell growth, migration, and angiogenesis in colon cancer cells and promotes the progression of colon cancer (9). LINC01123 promotes proliferation and metabolic rewiring in non-small cell lung cancer (NSCLC) cells and promotes tumor growth in vivo (10). However, to the best of our knowledge, the function of LINC01123 in CC has not been investigated.

MicroRNAs (miRNAs) are a type of short RNA molecule that play an important role in gene regulation (11). Previous studies show that certain miRNAs, such as miR-489 (12), miR-203 (13) and miR-338-3p are involved in CC proliferation (14). Recently, several studies have shown that miR-361-3p participates in CC progression. For example, low expression of miR-361-3p promotes cell proliferation and invasion in CC cells (15). Moreover, miR-361-3p inhibition enhances epithelial-mesenchymal transition, cell invasion and growth in CC cells (16).

In the present study, the expression of LINC01123, miR-361-3p and tetraspanin 1 (TSPAN1) was detected in $\mathrm{CC}$ tissue samples and cell lines. Thereafter, the regulatory effects of LINC01123 inhibition on cell viability, 
migration and invasion was surveyed in CC cells, as well as its effect on tumor growth in vivo. Furthermore, the mechanism by which LINC01123 knockdown regulated CC development via miR-361-3p/TSPAN1 was evaluated. The findings of the present study may present a novel target for CC treatment.

\section{Materials and methods}

Patient samples. Sixty-three patients with CC (63 women;

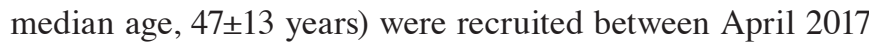
and June 2018 at Linyi Central Hospital (Linyi, China). None of the patients with $\mathrm{CC}$ underwent local or systemic treatment before surgery. CC tissue samples and corresponding adjacent healthy cervical tissue samples were acquired from the patients. All patients provided written informed consent and the study was approved by the Ethics Committee of Linyi Central Hospital (approval no. 2017013).

Cell culture and transfection. Human CC cell lines (HeLa, CaSki, SiHa and C-33A) and the human normal cervical epithelial cell line (HCerEpiC) were purchased from American Type Culture Collection. All cells were cultured in Gibco Dulbecco's modified Eagle's medium (DMEM; Thermo Fisher Scientific, Inc.) containing Gibco $10 \%$ fetal bovine serum (FBS; Thermo Fisher Scientific, Inc.) at $37^{\circ} \mathrm{C}$ in an incubator with $5 \% \mathrm{CO}_{2}$ until they were employed to perform the subsequent experiments.

Short hairpin RNA negative control (sh-NC, 5'-UUCUCC GAACGUGUCACGUTT-3'), sh-LINC01123-1 (5'-CUGAAC GUCUUGCAACAGUTT-3') and sh-LINC01123-2 (5'-GCC CUAGGAAAUCCGUAAUTT-3') were acquired from Sangon Biotech Co., Ltd. miRNA mimics NC (miR-NC, 5'-UUC UCCGAACGUGUCACGUTT-3'), miR-361-3p mimics (5'-UCCCCCAGGUGUGAUUCUGAUUU-3'), inhibitor NC (5'-UUCUCCGAACGUGUCACGUTT-3'), miR-361-3p inhibitor (5'-UCCCCCAGGUGUGAUUCUGAUUU-3'), as well as pcDNA-TSPAN1 and pcDNA-NC were purchased from GeneChem, Inc.. The above factors $(50 \mathrm{nM})$ were transfected into cells with Lipofectamine ${ }^{\circledR} 3000$ (Invitrogen; Thermo Fisher Scientific, Inc.). Cells were collected $48 \mathrm{~h}$ after transfection.

Reverse transcription-quantitative PCR (RT-qPCR) assay. According to the manufacturer's instructions, total RNA was extracted from tissue samples and cells using the TRIeasy Reagent kit (Qianchen Biotechnology Co., Ltd.). A Reverse Transcription Kit (Takara Bio, Inc.) was used according to the manufacturer's instructions to reverse transcribe RNA into cDNA. Thereafter, the expression levels of LINC01123, miR-361-3p and TSPAN1 were analyzed using the SYBR Green Real-Time PCR Kit (Takara Bio, Inc.) also according to the manufacturer's instructions. The expression levels of LINC01123 and TSPAN1 were normalized to GAPDH and U6 served as an endogenous control for miR-361-3p detection. RT-qPCR primer sequences were synthesized by Sangon Biotech Co., Ltd. and are presented in Table I. The PCR conditions were as follows: $95^{\circ} \mathrm{C}$ for $10 \mathrm{~min}$, followed by 40 cycles at $95^{\circ} \mathrm{C}$ for $30 \mathrm{sec}, 60^{\circ} \mathrm{C}$ for $30 \mathrm{sec}$ and $72^{\circ} \mathrm{C}$ for $1 \mathrm{~min}$. Data were calculated using the $2^{-\Delta \Delta \mathrm{Cq}}$ method (17).
3-(4,5-Dimethylthiazol-2-yl)-2,5-diphenyltetrazolium bromide (MTT) assay. Following transfection, cells were seeded in 96-well plates $\left(3 \times 10^{3}\right.$ cells/well) and incubated for $0,24,48,72$ and $96 \mathrm{~h}$ at $37^{\circ} \mathrm{C}$. Thereafter, $15 \mu \mathrm{l}$ MTT solution $(0.5 \mathrm{mg} / \mathrm{ml}$; Millipore Sigma) was added to each well. After 4 h, $200 \mu \mathrm{l}$ dimethyl sulfoxide was added to each well. The optical density of each well was read at $450 \mathrm{~nm}$ using a spectrophotometer (Thermo Fisher Scientific, Inc.).

Wound healing assay. The transfected cells were seeded in 6-well plates and grown to $100 \%$ confluence. The cells were scraped with a $10 \mu \mathrm{l}$ Eppendorf ${ }^{\mathrm{TM}}$ pipette tip and incubated for $24 \mathrm{~h}$ in a serum-free medium (Thermo Fisher Scientific, Inc.). Thereafter, they were washed using phosphate-buffered saline for three times to remove cell fragments and the lengths of the scratches were subsequently recorded using an Olympus Inverted Microscope. After $24 \mathrm{~h}$, the lengths of the scratches were recorded again. The healing rate was analyzed using ImageTool software (version 1.46, Los Alamos Operations). The wound closure was calculated as follows: (0-h width-24-h width)/0-h width $\mathrm{x} 100 \%$.

Transwell assay. To assess the invasion ability of cells, 24-well Transwell chambers (8.0 $\mu \mathrm{m}$; SproutStrong Biotech) were used to conduct the Transwell assay. Transfected cells $\left(2 \times 10^{5}\right.$ cells/well) in serum-free medium (Thermo Fisher Scientific, Inc.) were cultured in the upper compartment pre-coated with Matrigel (SproutStrong Biotech). The lower compartment contained DMEM with $15 \%$ FBS. After $24 \mathrm{~h}$, the cells in the lower compartment were fixed with $75 \%$ methanol at $4^{\circ} \mathrm{C}$ for $10 \mathrm{~min}$ and stained using $0.3 \%$ crystal violet at $37^{\circ} \mathrm{C}$ for $30 \mathrm{~min}$. Images were captured using an inverted optical microscope (Olympus).

Tumor formation assay in vivo. A total of 10 four-week-old BALB/c nude mice (female; weight 20-25 g) were obtained from Shanghai Laboratory Animal Centre (Shanghai, China). All mice were housed under controlled conditions $\left(25^{\circ} \mathrm{C}\right.$; $50 \%$ humidity; $12 \mathrm{~h}$ light/dark cycle) and were provided with free access to food and water. The mice were randomly divided into two groups of five mice. Lentiviral vectors (Lv) for sh-LINC01123-1 or sh-NC were constructed by Sangon Biotech Co., Ltd.. First, HeLa cells were transfected with Lv-sh-LINC01123-1 or Lv-sh-NC using Lipofectamine 3000. Thereafter, mice $(n=5)$ were injected with the HeLa cells $\left(1 \times 10^{6}\right.$; s.c.) in the right flank. Tumor volume (V) was measured at 10, 15, 20, 25 and 30 days after injection using the formula: $\mathrm{V}=$ length $\mathrm{x}$ width ${ }^{2} \mathrm{x} 0.5$. After 30 days, mice were anesthetized with pentobarbital sodium $(50 \mathrm{mg} / \mathrm{kg})$ and then sacrificed by cervical dislocation. The tumor xenograft was separated from the mice and weighed. The present study adhered to the requirements of the National Institutes of Health Guide for the Care and Use of Laboratory Animals and was approved by the Ethics Committee of Linyi Central Hospital (approval no. 2017013).

Bioinformatics analysis. The Cancer Genome Atlas (TCGA; https://www.cancer.gov/about-nci/organization/ ccg/research/structural-genomics/tcga) was used to examine LINC01123 expression in CC. 
Table I. Primers for reverse transcription-quantitative PCR.

\begin{tabular}{lll}
\hline Gene & \multicolumn{1}{c}{ Forward (5'-3') } & \multicolumn{1}{c}{ Reverse $\left(5^{\prime}-3^{\prime}\right)$} \\
\hline LINC01123 & ACAGTGGCCGCACGCATAGCTG & CTGACGACCGAGGTGACAACGATGA \\
miR-361-3p & ACACTCCAGCTGGGTCCCCCAG & CTCAACTGGTGTCGTGGAGTCGGCAA \\
TSPAN1 & GTGTGATTC & TTCAGTTGAGAAATCAGA \\
GAPDH & GTGGTCTTTGCTCTTGGTTTCC & TTTCTTGATGGCAGGCACTA \\
U6 & GCGAGATCGCACTCATCATCT & TCAGTGGTGGACCTGACC \\
\end{tabular}

LINC01123, long intergenic non-protein coding RNA 1123; miR, microRNA; TSPAN1, tetraspanin 1.

A

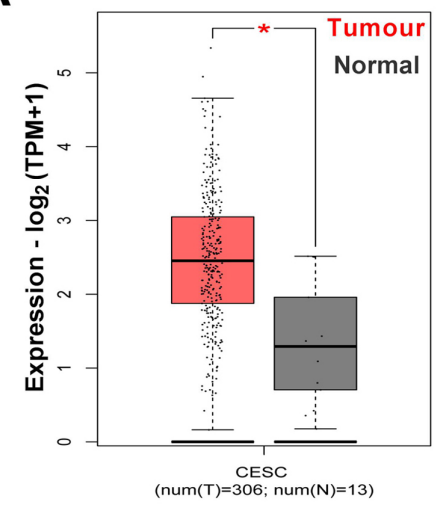

C

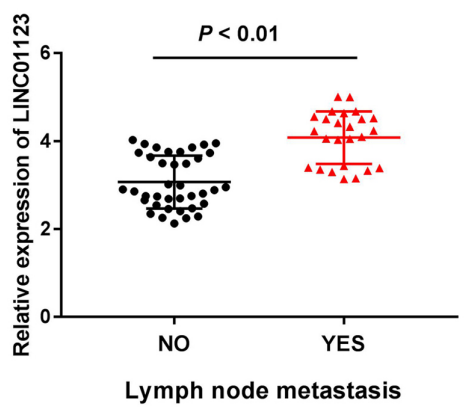

B

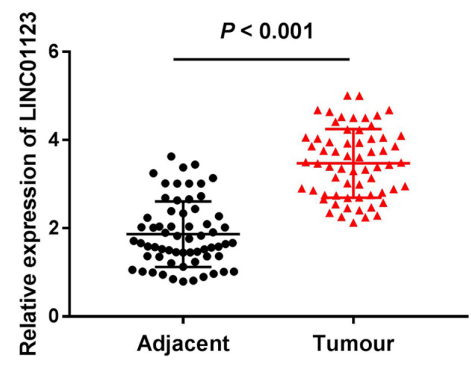

D

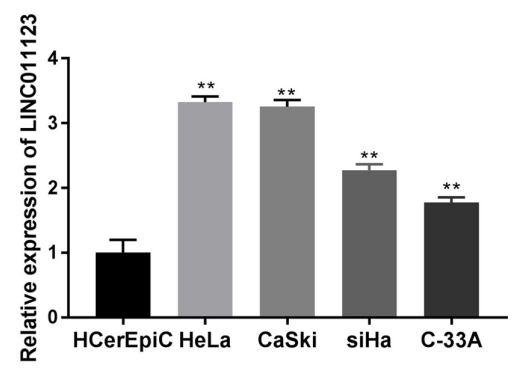

Figure 1. Expression of LINC01123 in CC tissue samples and cells. (A) The expression of LINC01123 was evaluated using The Cancer Genome Atlas database. ${ }^{*} \mathrm{P}<0.05$. (B) LINC01123 expression was detected by RT-qPCR in CC and adjacent healthy cervical tissue samples. P<0.001. (C) LINC01123 expression was detected by RT-qPCR in CC tissue samples with or without lymph node metastasis. $\mathrm{P}<0.01$. (D) LINC01123 expression was detected by RT-qPCR in CC cell lines (HeLa, CaSki, SiHa and C-33A) and normal cervical epithelial cell line (HCerEpiC). ${ }^{* *} \mathrm{P}<0.01$ vs. HCerEpiC. Experiments were performed in triplicate and repeated at least three times. LINC01123, long intergenic non-protein coding RNA 1123; CC, cervical cancer; RT-qPCR, reverse transcription-quantitative PCR; CESC, cervical squamous cell carcinoma and endocervical adenocarcinoma.

Dual-luciferase reporter (DLR) assay. The primary binding sites of LINC01123 and miR-361-3p, and miR-361-3p and TSPAN1 were predicted using LncBase Predicted v.2 (https://carolina.imis.athena-innovation.gr/diana_tools/web/ index.php? $\mathrm{r}=$ lncbasev2\%2Findex-predicted) and TargetScan (http://www.targetscan.org/vert_72/), respectively. The wild-type (WT) or mutant (MUT) binding sites of miR-361-3p to LINC01123 or TSPAN1 were inserted into a pGL3 vector (Promega Corporation) to generate corresponding luciferase reporters (LINC01123 WT, LINC01123 MUT, TSPAN1 WT and TSPAN1 MUT). Next, Lipofectamine 3000 was used to co-transfect the luciferase reporters and miR-361-3p mimics or miR-NC into HeLa and CaSki cells. Then, the relative luciferase activity was analyzed using a DLR ${ }^{\text {TM }}$ Assay System (Promega Corporation) after transfection for $24 \mathrm{~h}$.

Western blot analysis. HeLa and CaSki cells were lysed using radioimmunoprecipitation assay lysis solution (Beyotime Institute of Biotechnology). The protein concentration was detected by the BCA Protein Assay kit (Abcam). Protein samples (20 $\mu \mathrm{g}$ per lane) were separated equally with $10 \%$ sodium dodecyl sulphate-polyacrylamide gel electrophoresis and subsequently transferred to polyvinylidene difluoride membranes (EMD Millipore). The membranes were blocked with $5 \%$ non-fat milk for $1 \mathrm{~h}$ at $25^{\circ} \mathrm{C}$ and incubated overnight at $4^{\circ} \mathrm{C}$ with primary antibodies against TSPAN1 $(1: 1,000$; 
Table II. Correction of LINC01123 expression with clinicopathologic features in cervical cancer.

\begin{tabular}{|c|c|c|c|c|}
\hline \multirow[b]{2}{*}{ Characteristic } & \multirow[b]{2}{*}{$\mathrm{n}$} & \multicolumn{2}{|c|}{ LINC01123 expression } & \multirow[b]{2}{*}{ P-value } \\
\hline & & Low & High & \\
\hline Age (years) & & & & 0.693 \\
\hline$<45$ & 28 & 13 & 15 & \\
\hline$\geq 45$ & 35 & 18 & 17 & \\
\hline Menopause & & & & 0.537 \\
\hline No & 37 & 17 & 20 & \\
\hline Yes & 26 & 14 & 12 & \\
\hline Tumor size $(\mathrm{cm})$ & & & & 0.382 \\
\hline$<4$ & 25 & 14 & 11 & \\
\hline$\geq 4 \mathrm{~cm}$ & 38 & 17 & 21 & \\
\hline Depth of cervical invasion & & & & 0.098 \\
\hline$<2 / 3$ & 24 & 15 & 9 & \\
\hline$\geq 2 / 3$ & 39 & 16 & 23 & \\
\hline Histology & & & & 0.154 \\
\hline Squamous & 42 & 18 & 24 & \\
\hline Adenocarcinoma & 21 & 13 & 8 & \\
\hline Lymph node metastasis & & & & $0.027^{\mathrm{a}}$ \\
\hline No & 38 & 23 & 15 & \\
\hline Yes & 25 & 8 & 17 & \\
\hline FIGO stage & & & & $0.006^{\mathrm{a}}$ \\
\hline $\mathrm{I} / \mathrm{II}$ & 25 & 7 & 18 & \\
\hline III/IV & 38 & 24 & 14 & \\
\hline
\end{tabular}

${ }^{a} \mathrm{P}<0.05$. LINC01123, long intergenic non-protein coding RNA 1123; FIGO, International Federation of Gynecology and Obstetrics.

cat. no. ab254730) and $\alpha$-tubulin (1:1,000; cat. no. ab176560) purchased from Abcam. After the membranes were washed with TBST three times, the membranes were incubated at $37^{\circ} \mathrm{C}$ for $2 \mathrm{~h}$ with a horseradish peroxidase-conjugated secondary antibody (1:5,000; cat. no. ab205718) purchased from Abcam. The signals were determined using ECL Luminous Liquid (Pierce; Thermo Fisher Scientific, Inc.) and the immunoblots were analyzed using Image Lab ${ }^{\text {TM }}$ Software (Bio-Rad Laboratories, Inc.).

Statistical analysis. In vitro experiments were performed in triplicate and each experiment was repeated at least three times. In vivo experiments were performed using five mice in each of the two groups. Data analysis was performed using SPSS software version 20.0 (IBM Corp.) and data are presented as means \pm standard deviation (SD). Differences between two groups or among multiple groups were determined using Student's t-test or one-way ANOVA followed by Tukey's post-hoc test, respectively. Pearson's correlation analysis was also implemented to evaluate variate correlation. $\mathrm{P}<0.05$ was considered to indicate a statistically significant difference.

\section{Results}

LINC01123 expression is increased in CC tissue samples and cell lines. TCGA analysis demonstrated that LINC01123 expression was significantly increased in cervical squamous cell carcinoma and endocervical adenocarcinoma (CESC) tumors when compared to that in healthy tissue samples $(\mathrm{P}<0.05$; Fig. 1A). Furthermore, LINC01123 expression was highly enhanced in $\mathrm{CC}$ tissue samples when compared to that in adjacent healthy cervical tissue samples $(\mathrm{P}<0.001$; Fig. 1B). Meanwhile, LINC01123 expression was significantly enhanced in CC tissue samples with lymph node metastasis compared to that in tissue samples without metastasis $(\mathrm{P}<0.01$; Fig. $1 \mathrm{C})$. Furthermore, LINC01123 expression in CC cell lines (HeLa, CaSki, SiHa and C-33A) and the healthy cervical epithelial cell line (HCerEpiC) was examined. It was confirmed that LINC01123 expression was significantly enhanced in CC cell lines, particularly in CaSki and HeLa cells, when compared to that in HCerEpiC cells $(\mathrm{P}<0.01$; Fig. 1D). Thus, these two cells were selected for subsequent experiments. As presented in Table II, LINC01123 expression was significantly correlated with lymph node metastasis and International Federation of Gynecology and Obstetrics stage (18) $(\mathrm{P}<0.05)$. Thus, these data revealed that LINC01123 may contribute to CC tumorigenesis.

Knockdown of LINC01123 inhibits CC progression in vitro and in vivo. Based on the current findings, the biological function of LINC01123 in CC was investigated. Following transfection with sh-LINC01123-1 and sh-LINC01123-2, LINC01123 
$\mathbf{A}_{\mathscr{2}}$

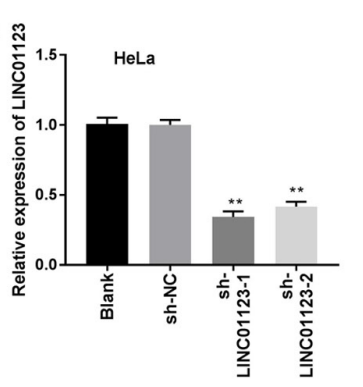

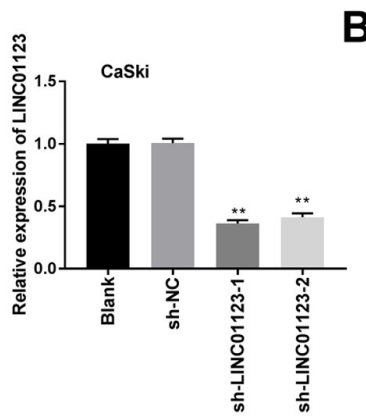

B
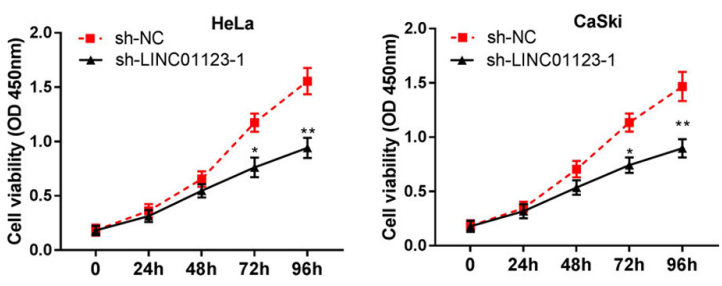

C
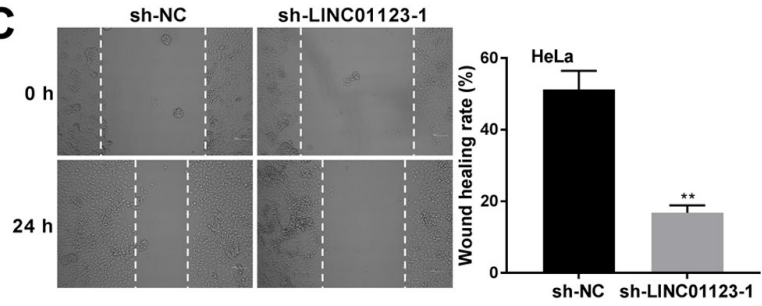

D
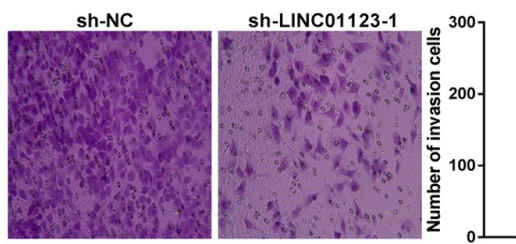

Hela

E

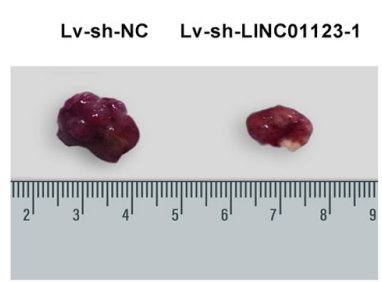

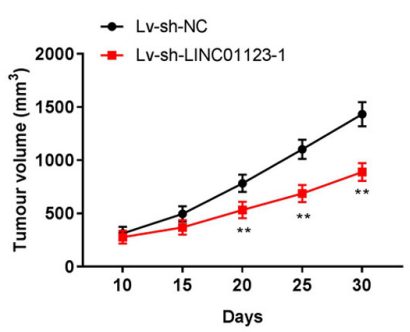

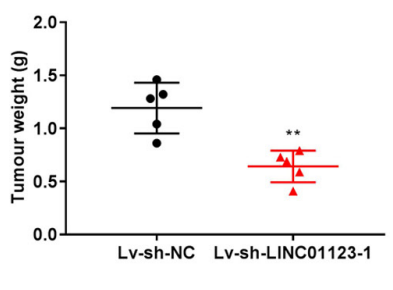

Figure 2. Silencing of LINC01123 inhibited CC progression in vitro and in vivo. (A) LINC01123 expression was detected by RT-qPCR in HeLa and CaSki cells transfected with sh-NC, sh-LINC01123-1 or sh-LINC01123-2. ${ }^{* *} \mathrm{P}<0.01$ vs. sh-NC. (B) Cell viability was detected by 3-(4,5-dimethylthiazol-2-yl)-2,5-diphenyltetrazolium bromide assay in HeLa and CaSki cells transfected with sh-LINC01123-1 or sh-NC. ${ }^{*} \mathrm{P}<0.05$ and ${ }^{* *} \mathrm{P}<0.01$ vs. sh-NC. (C) Cell migration was analyzed by wound healing assay in HeLa and CaSki cells transfected with sh-LINC01123-1 or sh-NC. ${ }^{* * *} \mathrm{P}<0.01$ vs. sh-NC. Magnification, $\mathrm{x} 400$. (D) Cell invasion was detected by Transwell assay in HeLa and CaSki cells transfected with sh-LINC01123-1 or sh-NC. ${ }^{* *} \mathrm{P}<0.01$ vs. sh-NC. The in vitro experiments were performed in triplicate and repeated at least three times. Magnification, x400. (E) Images, volume and weight change of tumors detected in the mouse xenograft models. ${ }^{* * *} \mathrm{P}<0.01$ vs. Lv-sh-NC. The in vivo experiments were performed on five mice in each group. LINC01123, long intergenic non-protein coding RNA 1123; CC, cervical cancer; RT-qPCR, reverse transcription-quantitative PCR; sh-NC, short hairpin RNA negative control; Lv, lentiviral vector.

expression in HeLa and CaSki cells was significantly reduced compared to that in the sh-NC group, and significantly greater inhibition of LINC01123 expression was observed in the sh-LINC1123-1 group than in the sh-LINC01123-2 group ( $\mathrm{P}<0.01$; Fig. 2A). Thus, sh-LINC01123-1 was selected for subsequent experiments. Thereafter, the knockdown of LINC01123 was observed to significantly inhibit cell viability in CaSki and HeLa cells ( $\mathrm{P}<0.01$; Fig. 2B). Furthermore, silencing LINC01123 notably suppressed cell invasion and migration in CaSki and HeLa cells $(\mathrm{P}<0.01$; Fig. $2 \mathrm{C}$ and $\mathrm{D})$. Meanwhile, the influence of LINC01123 knockdown on CC tumor growth in vivo was investigated by establishing mouse xenograft models. The volume and weight of $\mathrm{CC}$ tumors were found to be significantly decreased in the Lv-sh-LINC01123-1 group when compared to those in the Lv-sh-NC group $(\mathrm{P}<0.01$; Fig. 2E). Collectively, the results indicate that silencing LINC01123 inhibits CC cell viability, invasion and migration in vitro, and inhibits $\mathrm{CC}$ tumor growth in vivo.

miR-361-3p is a direct target of LINC01123. The primary target site between LINC01123 and miR-361-3p was predicted using LncBase Predicted v.2 (Fig. 3A). Knockdown of LINC01123 increased miR-361-3p expression in HeLa and CaSki cells $(\mathrm{P}<0.01$; Fig. 3B). Furthermore, the DLR assay demonstrated that miR-361-3p mimics significantly decreased the luciferase activity of LINC01123 WT in HeLa and CaSki cells $(\mathrm{P}<0.01$; Fig. 3C). Furthermore, miR-361-3p expression in CC tissue samples was lower than that in adjacent healthy cervical tissue 
A

\begin{tabular}{ccc} 
LINC01123 WT & AGGUC GGAUC & UGGGGGA \\
& $\mid$ | | | | | & |||||| $\mid$ \\
hsa-miR-361-3p & UUUAG CUUAG & ACCCCCU \\
& \multicolumn{2}{|c|}{ UGUGG }
\end{tabular}

LINC01123 MUT GAgGUCUgGaUCUgUgaugagaACcccCU

B

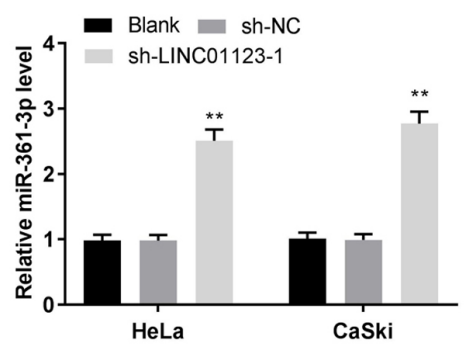

D

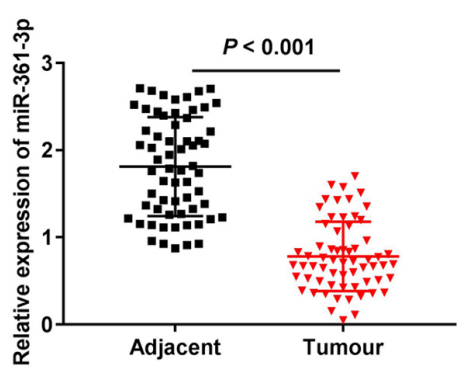

C

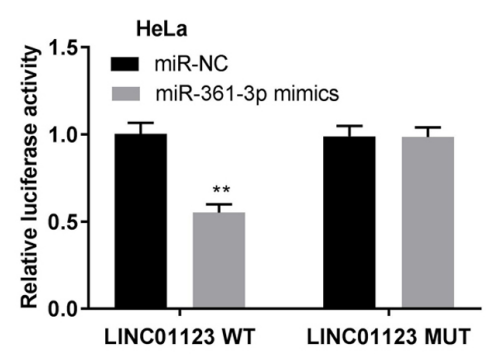

$\mathbf{E}$

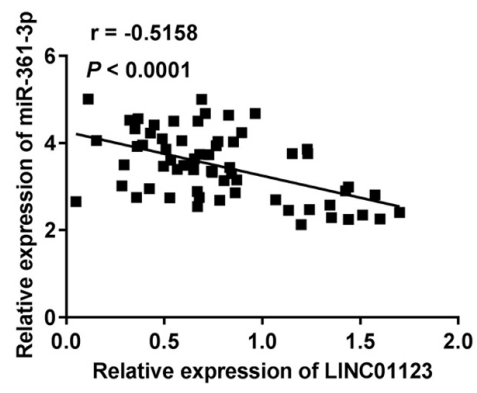

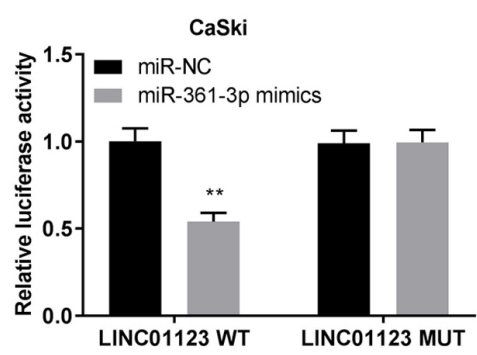

$\mathbf{F}$

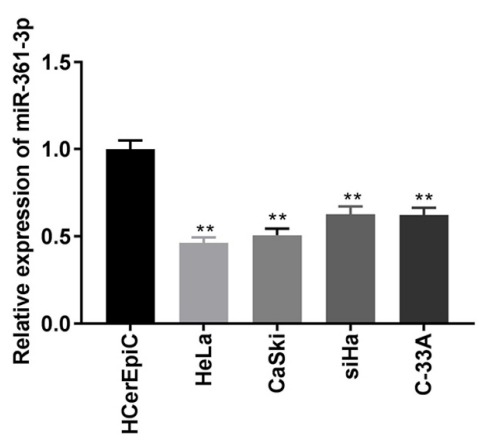

Figure 3. miR-361-3p acted as the direct target of LINC01123. (A) The possible binding site between LINC01123 and miR-361-3p was predicted by LncBase Predicted v.2. (B) The expression of miR-361-3p was detected by RT-qPCR in HeLa and CaSki cells transfected with sh-LINC01123-1 or sh-NC. ${ }^{* *}$ P $<0.01$ vs. sh-NC. (C) Dual-luciferase reporter assay confirmed the association between LINC01123 and miR-361-3p in HeLa and CaSki cells transfected with miR-NC, miR-361-3p mimics, LINC01123-WT or LINC01123-MUT. ${ }^{* *} \mathrm{P}<0.01$ vs. miR-NC. (D) miR-361-3p expression was detected by RT-qPCR in CC and adjacent healthy cervical tissue samples. $\mathrm{P}<0.001$. (E) The correlation between LINC01123 and miR-361-3p was evaluated by Pearson's correlation analysis. $\mathrm{P}<0.0001$. (F) The expression of miR-316-3p was detected by RT-qPCR in CC cell lines and HCerEpiC cells. ${ }^{* *} \mathrm{P}<0.01$ vs. HCerEpiC cells. The experiments were performed in triplicate and repeated at least three times. miR-361-3p, microRNA-361-3p; LINC01123, long intergenic non-protein coding RNA 1123; CC, cervical cancer; RT-qPCR, reverse transcription-quantitative PCR; sh-NC, short hairpin RNA negative control; WT, wild-type; MUT, mutant.

samples ( $\mathrm{P}<0.001$; Fig. 3D). Additionally, miR-361-3p expression was negatively correlated with LINC01123 expression in $\mathrm{CC}$ tissue samples $(\mathrm{r}=-0.5158 ; \mathrm{P}<0.0001)$ as shown in Fig. 3E. Thereafter, miR-361-3p expression in vitro was investigated. The results indicated that miR-361-3p expression in the CC cell lines was significantly reduced when compared to that in HCerEpiC cells $(\mathrm{P}<0.01$; Fig. 3F). Collectively, these results indicated that LINC01123 directly targeted miR-361-3p and negatively modulated miR-361-3p expression in CC.

miR-361-3p overexpression inhibits CC cell viability, migration and invasion. To elucidate the potential mechanism of miR-361-3p in CC progression, miR-361-3p mimics or miR-361-3p inhibitor were transfected into CaSki and HeLa cells. The results indicated that miR-361-3p expression was significantly increased in the miR-361-3p mimics group when compared to that in the miR-NC group, whereas miR-361-3p expression was decreased in the miR-361-3p inhibitor group when compared with the inhibitor NC group ( $\mathrm{P}<0.01$; Fig. $4 \mathrm{~A})$. Subsequently, miR-361-3p overexpression reduced cell viability in CaSki and HeLa cells ( $\mathrm{P}<0.01$; Fig. 4B). Similarly, cell migration and invasion in the miR-361-3p mimics group were significantly inhibited when compared to that in the miR-NC group ( $\mathrm{P}<0.01$; Fig. $4 \mathrm{C}$ and $\mathrm{D})$. Collectively, these results confirmed that miR-361-3p could inhibit cell viability, invasion and migration in CC cells.

TSPAN1 is a target of $m i R-361-3 p$. The primary target site between TSPAN1 and miR-361-3p was predicted using TargetScan (Fig. 5A). The results of the DLR assay indicated that miR-361-3p mimics significantly impaired luciferase activities of TSPAN1 WT in HeLa and CaSki cells $(\mathrm{P}<0.01$; Fig. 5B). Furthermore, the protein expression of TSPAN1 in HeLa and CaSki cells was suppressed by transfection of miR-361-3p mimics and sh-LINC01123-1 ( $\mathrm{P}<0.01$; Fig. 5C and D). TCGA analysis showed that TSPAN1 expression was significantly upregulated in CESC tumors compared to that in healthy tissues $(\mathrm{P}<0.05$; Fig. 5E). Additionally, the results from RT-qPCR confirmed that TSPAN1 expression was significantly enhanced in $\mathrm{CC}$ tissue samples when compared to that in adjacent healthy cervical tissue samples $(\mathrm{P}<0.001$; Fig. 5F). Furthermore, in $\mathrm{CC}$ tissue samples, 

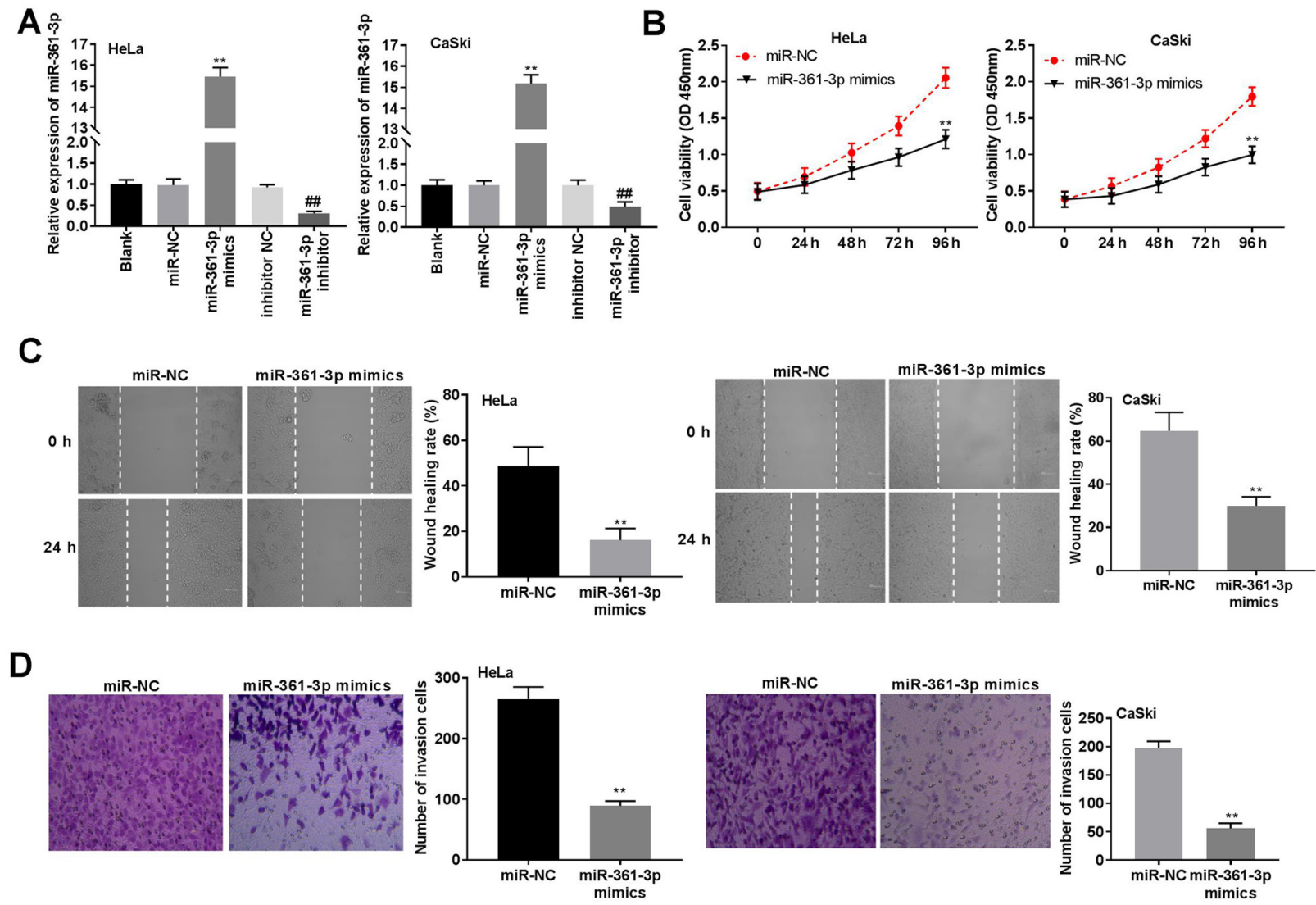

Figure 4. miR-361-3p overexpression inhibited CC cell viability, migration and invasion. (A) Expression of miR-361-3p was detected by RT-qPCR in HeLa and CaSki cells transfected with miR-361-3p mimics/miR-NC or miR-361-3p inhibitor/inhibitor NC. ${ }^{* *} \mathrm{P}<0.01$ vs. miR-NC, ${ }^{\# \#} \mathrm{P}<0.01 \mathrm{vs}$. inhibitor NC. (B) Cell viability was detected by 3-(4,5-dimethylthiazol-2-yl)-2,5-diphenyltetrazolium bromide assay in HeLa and CaSki cells transfected with miR-361-3p mimics or miR-NC. ${ }^{* *} \mathrm{P}<0.01$ vs. miR-NC. (C) Cell migration was analyzed by wound healing assay in HeLa and CaSki cells transfected with miR-361-3p mimics or miR-NC. ${ }^{* *} \mathrm{P}<0.01$ vs. miR-NC. Magnification, $\mathrm{x}$ 400. (D) Cell invasion was detected by Transwell assay in HeLa and CaSki cells transfected with miR-361-3p mimics or miR-NC. Magnification, $x 400 .{ }^{* *} \mathrm{P}<0.01$ vs. miR-NC. The experiments were performed in triplicate and repeated at least three times. miR-361-3p, microRNA-361-3p; CC, cervical cancer; $\mathrm{NC}$, negative control.

TSPAN1 expression was positively correlated with LINC01123 expression ( $\mathrm{r}=0.4455$; $\mathrm{P}=0.0003)$ as shown in Fig. $5 \mathrm{G}$ and was negatively correlated with miR-361-3p expression $(r=-0.4273$; $\mathrm{P}=0.0005)$ as shown in Fig. 5H. Collectively, these outcomes indicated that miR-361-3p targeted TSPAN1 and negatively regulated TSPAN1 expression in CC.

miR-361-3p interacts with TSPAN1 to mediate CC progression. To investigate the possible function of the miR-361-3p/TSPAN1 axis on CC progression in vitro, pcDNA-TSPAN1/NC was primarily transfected into HeLa cells to evaluate the protein expression of TSPAN1. As shown in Fig. 6A, the TSPAN1 expression level was elevated by pcDNA-TSPAN1 transfection $(\mathrm{P}<0.01)$. Using MTT, wound healing and Transwell assays, the inhibitory effects of miR-361-3p mimics on viability, migration and invasion of HeLa cells were observed to be ameliorated by pcDNA-TSPAN1 ( $\mathrm{P}<0.01$; Fig. 6B-D).

LINC01123 regulates CC tumorigenesis by targeting miR-361-3p/TSPAN1. Whether the tumor-suppressive effect of LINC01123 knockdown was dependent on miR-361-3p and TSPAN1 in CC progression was subsequently investigated. As demonstrated in Fig. 7A, LINC01123 knockdown decreased HeLa cell viability, while miR-361-3p inhibition or TSPAN1 overexpression ameliorated this trend $(\mathrm{P}<0.01)$. Furthermore, silencing LINC01123 inhibited the migration and invasion of HeLa cells, while miR-361-3p inhibition or TSPAN1 overexpression was found to ameliorate the inhibitory effect $(\mathrm{P}<0.01$; Fig. 7B and $\mathrm{C})$. These data demonstrated that the silencing of LINC01123 inhibited CC tumorigenesis via miR-361-3p/TSPAN1 interaction.

\section{Discussion}

$\mathrm{CC}$ is a gynecological malignancy, which poses an enormous threat to females (19). Thus, identifying available targets to improve prognosis and effectively treat $\mathrm{CC}$ is considered critical. Numerous 1 ch RAs have been reported to play vital roles in $\mathrm{CC}$ tumorigenesis $(20,21)$. In the current study, LINC01123 expression was enhanced in CC tissue samples and cell lines. Silencing of LINC01123 inhibited cell viability, migration and invasion in vitro and suppressed tumor growth in vivo. Furthermore, LINC01123 negatively regulated miR-361-3p and miR-361-3p negatively regulated TSPAN1. Further experiments demonstrated that LINC01123 knockdown regulated the progression of $\mathrm{CC}$ by targeting miR-361-3p/TSPAN1; thus, it could likely serve as a primary target for the treatment of CC. 

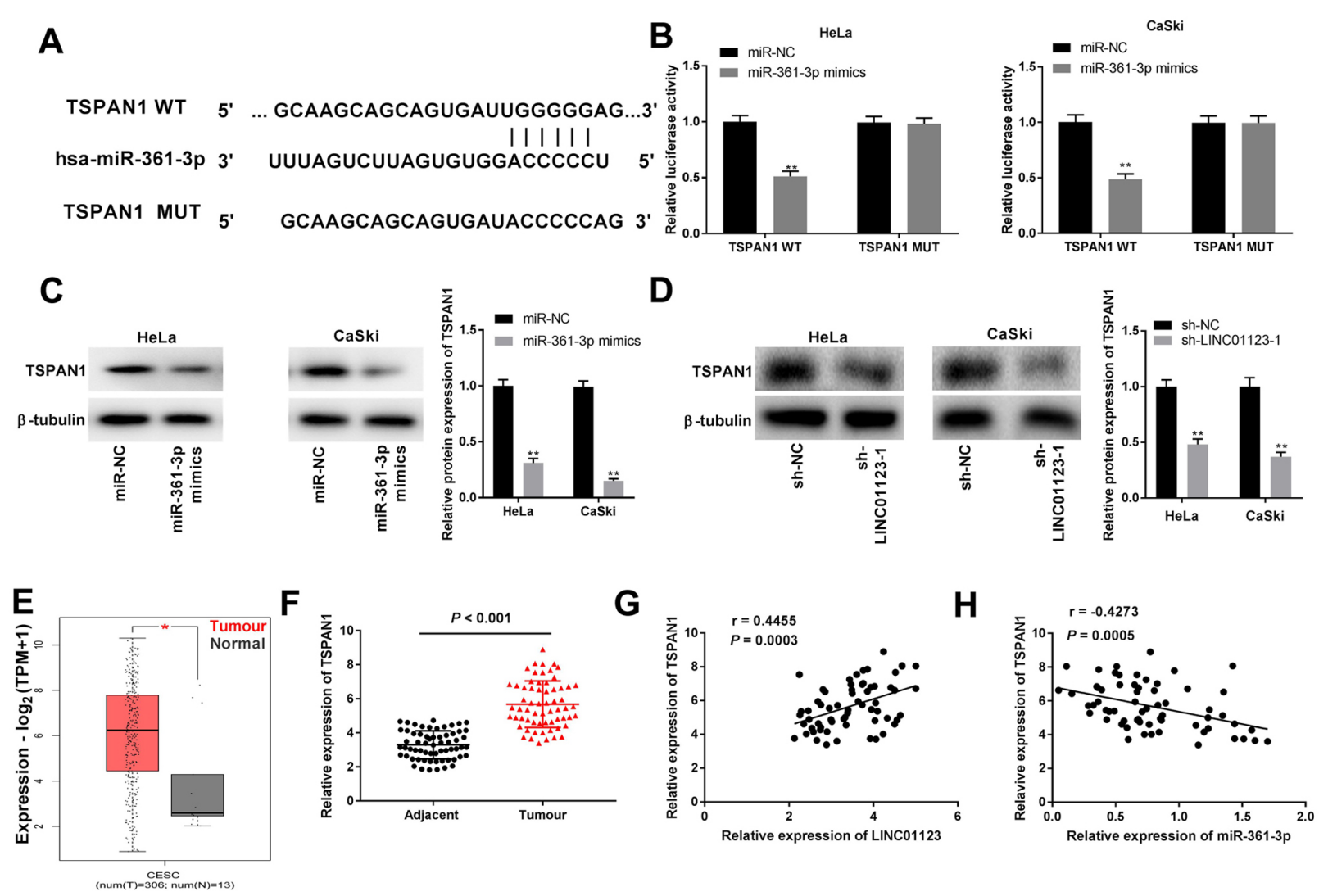

Figure 5. TSPAN1 was the direct target gene of miR-361-3p. (A) The putative binding site of miR-361-3p was predicted by TargetScan. (B) Dual-luciferase reporter assay confirmed the association between miR-361-3p and TSPAN1 in HeLa and CaSki cells transfected with miR-NC, miR-361-3p mimics, TSPAN1 WT or TSPAN1 MUT. * P $<0.01$ vs. miR-NC. (C) The expression of TSPAN1 protein was detected by western blotting in HeLa and CaSki cells transfected with miR-NC and miR-361-3p mimics. ${ }^{* * *} \mathrm{P}<0.01$ vs. miR-NC. (D) The expression of TSPAN1 protein was detected by western blotting in HeLa and CaSki cells transfected with sh-NC and sh-LINC01123-1. ${ }^{* *} \mathrm{P}<0.01$ vs. sh-NC. (E) Relative expression of TSPAN1 was evaluated by The Cancer Genome Atlas database. ${ }^{*} \mathrm{P}<0.05$. (F) TSPAN1 expression was detected by reverse transcription-quantitative PCR in CC and adjacent healthy cervical tissue samples. $\mathrm{P}<0.001$. The correlation between (G) TSPAN1 and LINC01123 ( $\mathrm{P}=0.0003)$ and (H) TSPAN1 and miR-361-3p ( $\mathrm{P}=0.0005)$ was evaluated by Pearson's correlation analysis. The experiments were performed in triplicate and repeated at least three times. TSPAN1, tetraspanin 1; miR-361-3p, microRNA-361-3p; CC, cervical cancer; NC, negative control; WT, wild-type; MUT, mutant; sh, short hairpin RNA; LINC01123, long intergenic non-protein coding RNA 1123.

LINC01123 is a lncRNA activated by c-Myc, which exerts oncogenic effects in certain types of cancer. Previous studies show that LINC01123 is upregulated in colon cancer (9), NSCLC (10) and triple-negative breast cancer (22). In the present study, LINC01123 expression was also increased in CC tissue samples and cell lines, suggesting its association with CC development. Numerous studies show that LINC01123 promotes cell growth, invasion and migration in several cancer cell lines, such as colon (9) and endometrial (23) cancer cells. Similarly, the present results demonstrated that silencing LINC01123 inhibited cell viability, migration and invasion in HeLa and CaSki cells, indicating that LINC01123 knockdown has a tumor-suppressive effect in vitro. To further verify the inhibitory function of LINC01123 knockdown on CC tumor growth in vivo, mouse xenograft models were established. Results showed that the knockdown of LINC01123 suppressed the volume and weight of CC tumors in vivo. These results indicated that silencing of LINC01123 plays a vital role in inhibiting CC development.

Previous studies have reported that miR-361-3p participates in the progression of several types of cancer, including colorectal cancer (CRC) (24), thyroid cancer (25) and CC (16) and its expression is downregulated in these cancer types. Similar to the above results $(16,24,25)$, the present study showed that miR-361-3p expression was also downregulated in
CC tissue samples and cell lines, suggesting that miR-361-3p may be a tumor-suppressor gene in CC. LncRNAs can act as competing endogenous RNAs to exert their functions by targeting miRNAs (26). LINC01123 facilitates cell proliferation and tumor growth in NSCLC by targeting miR-199a-5p (10). LINC01123 facilitates the proliferation and invasion of CRC cells via miR-625-5p sponging (27). Notably, both LINC00460 and BBOX1 antisense RNA 1 interact with miR-361-3p to affect CC progression $(24,28)$. In the current study, LINC01123 directly targeted miR-361-3p and negatively modulated miR-361-3p expression in CC tissue samples. In addition, overexpression of miR-361-3p inhibits cell growth and invasion in NSCLC (29) and HeLa (16) cells.

In the present study, overexpression of miR-361-3p was observed to inhibit cell viability, invasion and migration in $\mathrm{HeLa}$ and CaSki cells. The present study further hypothesized that LINC01123 silencing may ameliorate the malignant behavior of CC by regulating miR-361-3p. TSPAN1, a transmembrane protein, is involved in cancer progression (30). Previous studies have confirmed that TSPAN1 is targeted by miR-491-3p in osteosarcoma (31) and miR-216a in pancreatic cancer (32). In the present study, TSPAN1 was found to be targeted by miR-361-3p and its expression was negatively modulated by miR-361-3p in CC. It has been reported that TSPAN1 expression is upregulated 
A

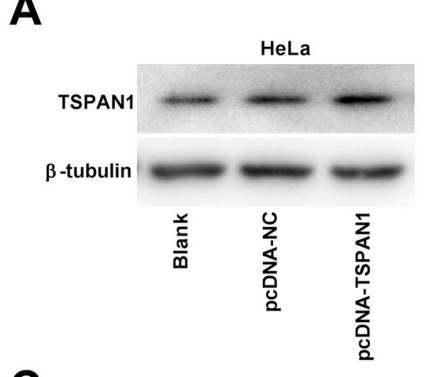

C

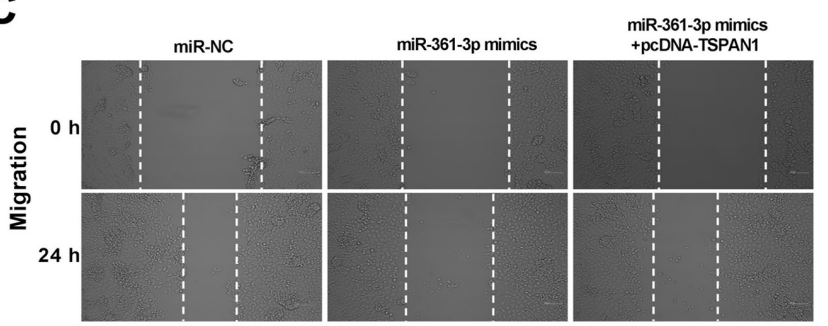

D
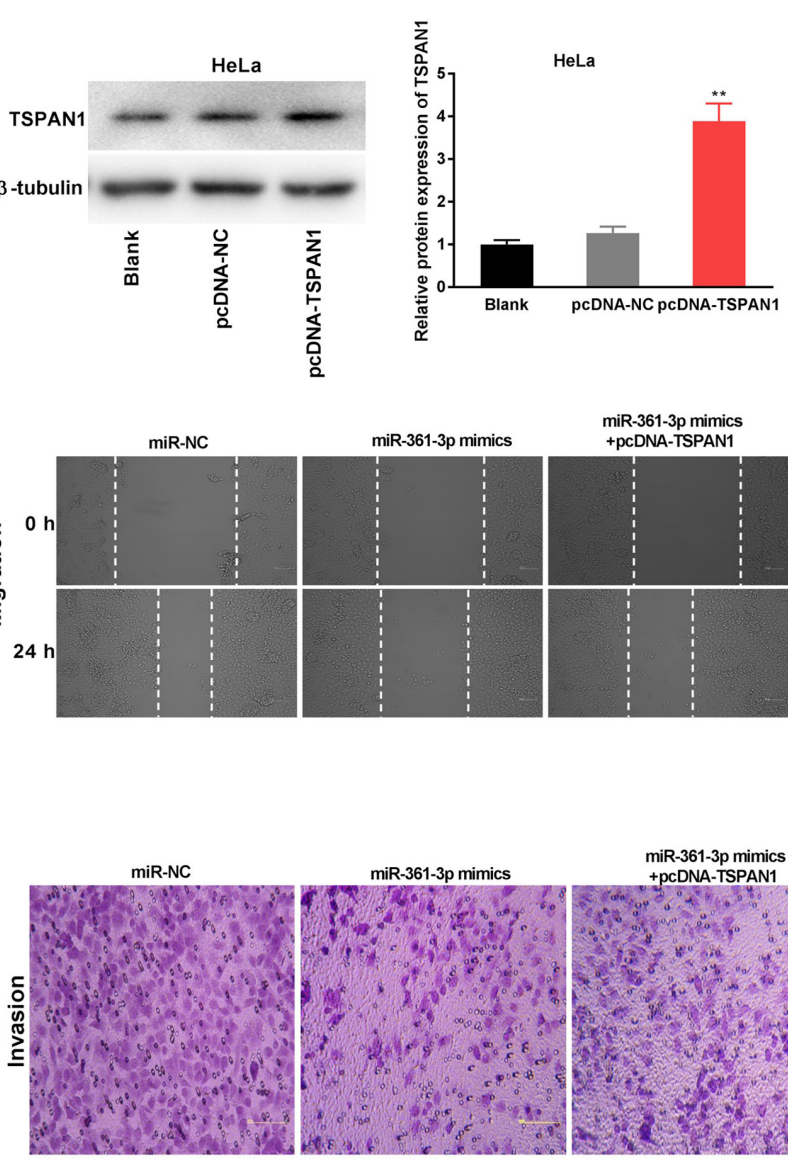

B
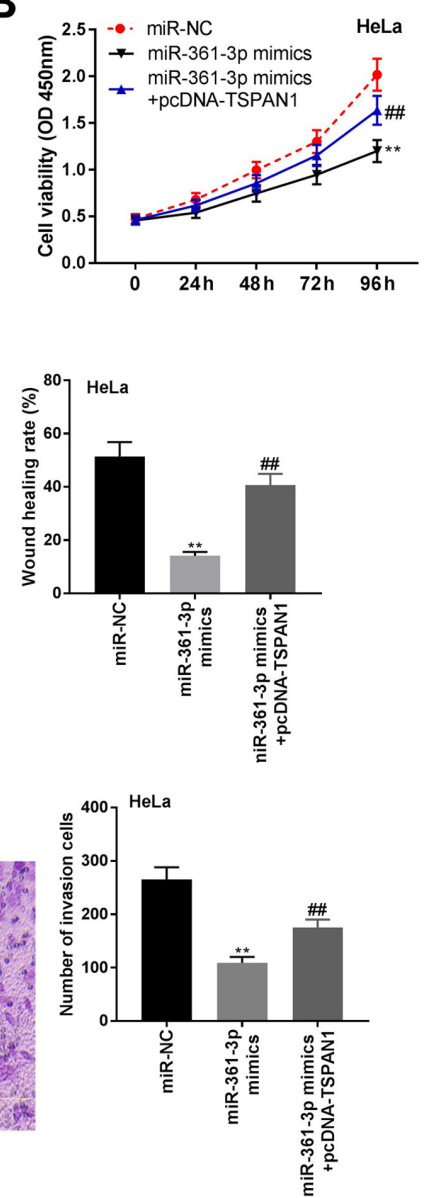

Figure 6. miR-361-3p interacted with TSPAN1 to mediate CC progression. (A) The expression of TSPAN1 protein was detected by western blotting in HeLa cells transfected with pcDNA-NC or pcDNA-TSPAN1. * P $<0.01$ vs. pcDNA-NC. (B) Cell viability was detected by 3-(4,5-dimethylthiazol-2-yl)-2,5-diphenyltetrazolium bromide assay in HeLa cells transfected with miR-NC, miR-361-3p mimics or miR-361-3p mimics + pcDNA-TSPAN1. * P<0.01 vs. miR-NC. ${ }^{\# \prime} \mathrm{P}<0.01$ vs. miR-361-3p mimics. (C) Cell migration was analyzed by wound healing assay in HeLa cells transfected with miR-NC, miR-361-3p mimics or miR-361-3p mimics + pcDNA-TSPAN1. ${ }^{* *} \mathrm{P}<0.01$ vs. miR-NC. ${ }^{\# \#} \mathrm{P}<0.01$ vs. miR-361-3p mimics. Magnification, $\mathrm{x} 400$. (D) Cell invasion was detected by Transwell assay in HeLa cells transfected with miR-NC, miR-361-3p mimics or miR-361-3p mimics + pcDNA-TSPAN1. ${ }^{* *} \mathrm{P}<0.01 \mathrm{vs}$. miR-NC. ${ }^{\# \#} \mathrm{P}<0.01 \mathrm{vs}$. miR-361-3p mimics. Magnification, x400. The experiments were performed in triplicate and repeated at least three times. TSPAN1, tetraspanin 1; miR-361-3p, microRNA-361-3p; CC, cervical cancer; NC, negative control. magnification x400.
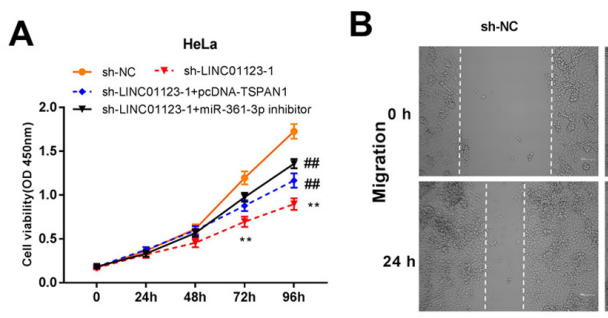

sh-LINC01123-1
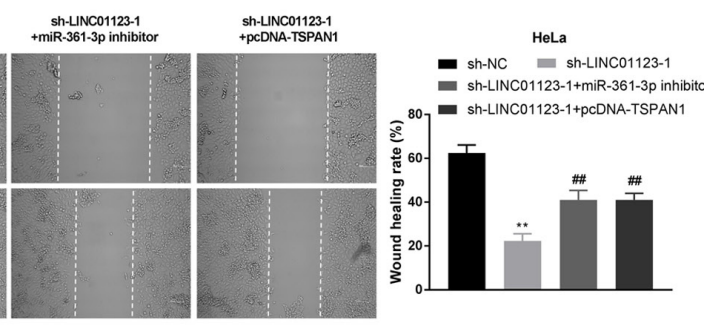

C

sh-NC

sh-LINC01123-1

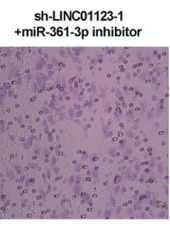

HeLa
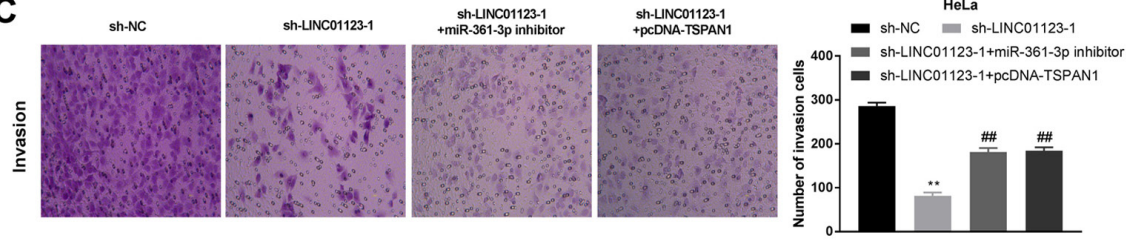

Figure 7. LINC01123 regulated tumorigenesis of CC by targeting miR-361-3p/TSPAN1. (A) Cell viability was detected by 3-(4,5-dimethylthiazol-2-yl)-2,5-diphenyltetrazolium bromide assay in HeLa cells transfected with sh-NC, sh-LINC01123-1, sh-LINC01123-1 + pcDNA-TSPAN1 or sh-LINC01123-1 + miR-361-3p. ${ }^{* *} \mathrm{P}<0.01$ vs. sh-NC. ${ }^{\# \#} \mathrm{P}<0.01$ vs. sh-LINC01123-1. (B) Cell migration was analyzed by wound healing assay in HeLa cells transfected with sh-NC, sh-LINC01123-1, sh-LINC01123-1 + pcDNA-TSPAN1 or sh-LINC01123-1 + miR-361-3p. ${ }^{* *} \mathrm{P}<0.01$ vs. sh-NC. ${ }^{\sharp \# P} \mathrm{P}<0.01$ vs. sh-LINC01123-1. Magnification, x400. (C) Cell invasion was detected by Transwell assay in HeLa cells transfected with sh-NC, sh-LINC01123-1, sh-LINC01123-1 + pcDNA-TSPAN1 or sh-LINC01123-1 + miR-361-3p. ${ }^{* *} \mathrm{P}<0.01$ vs. sh-NC. ${ }^{\# \#} \mathrm{P}<0.01$ vs. sh-LINC01123-1. Magnification, $\mathrm{x} 400$. The experiments were performed in triplicate and repeated at least three times. LINC01123, long intergenic non-protein coding RNA 1123; CC, cervical cancer; miR-361-3p, microRNA-361-3p; TSPAN1, tetraspanin 1; NC, negative control; sh, short hairpin RNA. 
in gastric carcinoma (33), ovarian carcinoma (34), pancreatic cancer (35) and CC (36). Similarly, the present study found that TSPAN1 expression was also upregulated in $\mathrm{CC}$ tissue samples, indicating that TSPAN1 may be an oncogene in CC.

Previous studies have shown that TSPAN1 increases cell growth and invasion in certain types of cancer. For example, the overexpression of TSPAN1 enhances cell invasion in CC cell lines (36). In addition, miR-573 overexpression inhibits cell viability and invasion by decreasing TSPAN1 expression in gastric cancer (37). Thus, it was hypothesized that miR-361-3p might regulate cell viability, migration and invasion by regulating TSPAN1 in CC cells. As LINC01123 directly targeted miR-361-3p, it was hypothesized that LINC01123 might facilitate CC tumorigenesis by regulating the miR-361-3p/TSPAN1 axis. Further rescue studies confirmed that miR-361-3p inhibition and TSPAN1 overexpression reversed LINC01123 knockdown-induced inhibition of cell viability, migration and invasion in HeLa cells, indicating that LINC01123 knockdown inhibited CC tumorigenesis by targeting miR-361-3p/TSPAN1.

Thus, the present study revealed that LINC01123 expression was increased in $\mathrm{CC}$ tissue samples and cell lines. Silencing of LINC01123 inhibited cell viability, invasion and migration in vitro and suppressed tumor growth in vivo. miR-361-3p was targeted by LINC01123, whereas TSPAN1 was targeted by miR-361-3p. Furthermore, knockdown of LINC01123 inhibited CC tumorigenesis by regulating the miR-361-3p/TSPAN1 axis. However, there may be some upstream factors or related signaling pathways interaction with the LINC01123/miR-361-3p/TSPAN1 axis in the progression of CC. This is a direction for future studies. However, the findings of the present study may also provide valuable information in the therapy of $\mathrm{CC}$.

\section{Acknowledgements}

Not applicable.

\section{Funding}

No funding was received.

\section{Availability of data and materials}

All data generated or analyzed during this study are included in this published article.

\section{Authors' contributions}

$\mathrm{CL}$ and $\mathrm{CH}$ made substantial contributions to the conception and design of the study. CL, YL, YZ and HY made substantial contributions to the acquisition, analysis and interpretation of data, as well as the drafting and revision of the manuscript. CL, YL, YZ HY and CH confirm the authenticity of all the raw data and approved the final manuscript. All authors read and approved the final manuscript.

\section{Ethics approval and consent to participate}

All patients provided signed informed consent and the Ethics Committee of Linyi Central Hospital approved the study. (approval no. 2017013). Animal experiments were performed according to the National Institutes of Health Guide for the Care and Use of Laboratory Animals (approval no. 2017013).

\section{Patient consent for publication}

Not applicable.

\section{Competing interests}

The authors declare that they have no competing interests.

\section{References}

1. Morris E and Roett MA: Genital cancers in Women: Cervical cancer. FP Essent 438: 18-23, 2015.

2. Arbyn M, Weiderpass E, Bruni L, de Sanjosé S, Saraiya M, Ferlay J and Bray F: Estimates of incidence and mortality of cervical cancer in 2018: A worldwide analysis. Lancet Glob Health 8: e191-e203, 2020.

3. Kori M and Yalcin Arga K: Potential biomarkers and therapeutic targets in cervical cancer: Insights from the meta-analysis of transcriptomics data within network biomedicine perspective. PLoS One 13: e0200717, 2018.

4. $\mathrm{Li} \mathrm{H}, \mathrm{Wu} \mathrm{X}$ and Cheng X: Advances in diagnosis and treatment of metastatic cervical cancer. J Gynecol Oncol 27: e43, 2016.

5. Yee GP, de Souza P and Khachigian LM: Current and potential treatments for cervical cancer. Curr Cancer Drug Targets 13: 205-220, 2013.

6. Sanchez Calle A, Kawamura Y, Yamamoto Y, Takeshita F and Ochiya T: Emerging roles of long non-coding RNA in cancer. Cancer Sci 109: 2093-2100, 2018.

7. Luan X and Wang Y: LncRNA XLOC_006390 facilitates cervical cancer tumorigenesis and metastasis as a ceRNA against miR-331-3p and miR-338-3p. J Gynecol Oncol 29: e95, 2018.

8. Liu Y, Yang Y, Li L, Liu Y, Geng P, Li G and Song H: LncRNA SNHG1 enhances cell proliferation, migration, and invasion in cervical cancer. Biochem Cell Biol 96: 38-43, 2018.

9. Ye S, Sun B, Wu W, Yu C, Tian T, Lian Z, Liang Q and Zhou Y: LINC01123 facilitates proliferation, invasion and chemoresistance of colon cancer cells. Biosci Rep 40: BSR20194062, 2020.

10. Hua Q, Jin M, Mi B, Xu F, Li T, Zhao L, Liu J and Huang G: LINC01123, a c-Myc-activated long non-coding RNA, promotes proliferation and aerobic glycolysis of non-small cell lung cancer through miR-199a-5p/c-Myc axis. J Hematol Oncol 12: 91, 2019.

11. Bartel DP: MicroRNAs: Genomics, biogenesis, mechanism, and function. Cell 116: 281-297, 2004.

12. Juan C, Hua Q, Ruping Z and Tingting W: miRNA-489 as a biomarker in diagnosis and treatment of cervical cancer. Bratisl Lek Listy 119: 278-283, 2018

13. Yin XZ, Zhao DM, Zhang GX and Liu L: Effect of miRNA-203 on cervical cancer cells and its underlying mechanism. Genet Mol Res 15, 2016.

14. Hua FF, Liu SS, Zhu LH, Wang YH, Liang X, Ma N and Shi HR: MiRNA-338-3p regulates cervical cancer cells proliferation by targeting MACC1 through MAPK signaling pathway. Eur Rev Med Pharmacol Sci 21: 5342-5352, 2017.

15. Liu S, Song L, Yao H, Zhang L, Xu D, Li Q and Li Y: Preserved miR-361-3p Expression is an independent prognostic indicator of favorable survival in cervical cancer. Dis Markers 2018: 8949606, 2018.

16. Wang J, Li H and Liang Z: circ-MYBL2 serves as a sponge for miR-361-3p promoting cervical cancer cells proliferation and invasion. Onco Targets Ther 12: 9957-9964, 2019.

17. Livak KJ and Schmittgen TD: Analysis of relative gene expression data using real-time quantitative PCR and the 2(-Delta Delta C(T)) method. Methods 25: 402-408, 2001.

18. Matsuo K, Machida H, Mandelbaum RS, Konishi I and Mikami M: Validation of the 2018 FIGO cervical cancer staging system. Gynecol Oncol 152: 97-93, 2019.

19. Tewari KS, Sill MW, Long HJ III, Penson RT, Huang H, Ramondetta LM, Landrum LM, Oaknin A, Reid TJ, Leitao MM, et al: Improved survival with bevacizumab in advanced cervical cancer. N Engl J Med 370: 734-743, 2014. 
20. Hsu W, Liu L, Chen X, Zhang Y and Zhu W: LncRNA CASC11 promotes the cervical cancer progression by activating Wnt/beta-catenin signaling pathway. Biol Res 52: 33, 2019.

21. Wang R,Li Y,Du P,Zhang X,Li X and Cheng G: Hypomethylation of the lncRNA SOX21-AS1 has clinical prognostic value in cervical cancer. Life Sci 233: 116708, 2019.

22. Zhang P, Long Q, Zeng S, Wen M and Lu Q: FOXC1-induced LINC01123 acts as a mediator in triple negative breast cancer. Cancer Cell Int 20: 199, 2020.

23. Yang Y, Wu J,Zhou H,Liu W, Wang J and Zhang Q: STAT1-induced upregulation of lncRNA LINC01123 predicts poor prognosis and promotes the progression of endometrial cancer through miR-516b/KIF4A. Cell Cycle 19: 1502-1516, 2020.

24. Liu J, Zhu J, Xiao Z, Wang X and Luo J: BBOX1-AS1 contributes to colorectal cancer progression by sponging hsa-miR-361-3p and targeting SH2B1. FEBS Open Bio, Jan 27, 2020 (Epub ahead of print).

25. Xia F, Chen Y, Jiang B, Bai N and Li X: Hsa circ 0011385 accelerates the progression of thyroid cancer by targeting miR-361-3p. Cancer Cell Int 20: 49, 2020.

26. Tay Y, Rinn J and Pandolfi PP: The multilayered complexity of ceRNA crosstalk and competition. Nature 505: 344-352, 2014

27. Shang T, Zhou $X$ and Chen W: LINC01123 promotes progression of colorectal cancer via miR-625-5p/LASP1 axis. Cancer Biother Radiopharm, May 18, 2020 (Epub ahead of print).

28. Li F, Zhu W and Wang Z: Long noncoding RNA LINC00460 promotes the progression of cervical cancer via regulation of the miR-361-3p/Gli1 axis. Hum Cell 34: 229-237, 2021.

29. Chen W, Wang J, Liu S, Wang S, Cheng Y, Zhou W, Duan C and Zhang C: MicroRNA-361-3p suppresses tumor cell proliferation and metastasis by directly targeting SH2B1 in NSCLC. J Exp Clin Cancer Res 35: 76, 2016.
30. Huang S, Yuan S, Dong M, Su J, Yu C, Shen Y, Xie X, Yu Y, Yu X, Chen S, et al: The phylogenetic analysis of tetraspanins projects the evolution of cell-cell interactions from unicellular to multicellular organisms. Genomics 86: 674-684, 2005.

31. Duan J, Liu J, Liu Y, Huang B and Rao L: miR-491-3p suppresses the growth and invasion of osteosarcoma cells by targeting TSPAN1. Mol Med Rep 16: 5568-5574, 2017.

32. Wang S, Liu X, Khan AA, Li H, Tahir M, Yan X, Wang J and Huang $\mathrm{H}$ : miR-216a-mediated upregulation of TSPAN1 contributes to pancreatic cancer progression via transcriptional regulation of ITGA2. Am J Cancer Res 10: 1115-1129, 2020.

33. Chen L, Li X, Wang GL, Wang Y, Zhu YY and Zhu J: Clinicopathological significance of overexpression of TSPAN1, Ki67 and CD34 in gastric carcinoma. Tumori 94: 531-538, 2008.

34. Scholz CJ, Kurzeder C, Koretz K, Windisch J, Kreienberg R, Sauer G and Deissler H: Tspan-1 is a tetraspanin preferentially expressed by mucinous and endometrioid subtypes of human ovarian carcinomas. Cancer Lett 275: 198-203, 2009.

35. Tian J, Zhang R, Piao H, Li X, Sheng W, Zhou J, Dong M, Zhang X, Yan X, Shang W, et al: Silencing Tspan1 inhibits migration and invasion, and induces the apoptosis of human pancreatic cancer cells. Mol Med Rep 18: 3280-3288, 2018.

36. Hölters S, Anacker J, Jansen L, Beer-Grondke K, Dürst M and Rubio I: Tetraspanin 1 promotes invasiveness of cervical cancer cells. Int J Oncol 43: 503-512, 2013.

37. Lu Z, Luo T, Nie M, Pang T, Zhang X, Shen X, Ma L, Bi J, Wei G, Fang G and Xue X: TSPAN1 functions as an oncogene in gastric cancer and is downregulated by miR-573. FEBS Lett 589: 1988-1994, 2015 